\title{
Patrimonio biocultural de Tabasco: elaboración de platillos tradicionales en comunidades indígenas
}

\section{Tabasco biocultural heritage: making traditional dishes in indigenous communities}

CARRILLO-CONTRERAS, Jaime†, MAY-ARIAS, Elsi Margarita y MAZÓN-PÉREZ, Silvia Gertrudis

ID $1^{\text {er }}$ Autor: Jaime, Carrillo-Contreras

ID $1^{\mathrm{er}}$ Coautor: Elsi Margarita, May-Arias

ID $2^{\text {do }}$ Coautor: Silvia Gertrudis, Mazón-Pérez.

DOI: $10.35429 / J O C S .2019 .19 .6 .7 .15$

Recibido: 18 de Abril, 2019; Aceptado 28 de Junio, 2019

\section{Resumen}

El objetivo de este trabajo fue Identificar los platillos tradicionales en los municipios de Centla y Tacotalpa, Tabasco; para reconocer el patrimonio biocultural en zonas indígenas. Esta investigación se desarrolló desde el año 2016 hasta el año 2018. La metodología utilizada fue cualitativa, mediante la aplicación de entrevistas abiertas a cocineras o cocineros tradicionales; la selección de personas se realizó con los familiares o conocidos de los estudiantes de la licenciatura en Desarrollo Turístico. Las entrevistas fueron grabadas en un dispositivo móvil y posteriormente se transcribieron a un procesador de texto; finalmente la información generada se agregó a una base de datos. Los resultados obtenidos muestran que se identificó un total de 69 platillos, distribuidos para el municipio de Centla (40) y en Tacotalpa (29); con respecto a los nombres de los platillos en lengua indígena, se identificaron solo 2 en lengua Zoque para el municipio de Tacotalpa. La relación entre los recursos naturales y el patrimonio biocultural para Centla se encontró principalmente con grupos de fauna como Crustáceos, Quelonios, Reptiles y Peces; y en la zona Serrana de Tacotalpa por Plantas, Mamíferos, Gasterópodos y Peces.

\begin{abstract}
The objective about this research was to identify the traditional dishes in the municipality from Centla and Tacotalpa that belongs to the territory of Tabasco for recognizing the biocultural heritage at indigenous areas. This survey was developed since 2016 to 2018 and the way of study and methodology was cualitative through the open interviews to people who cooks traditional dishes in order to have a selection of chefs were in charge the plates. This selection was performed with persons related to the students of turistic development career in this case families, relatives and acquaintancies. The interviews were recorded in a mobile divice and transcribed to a Word processor in order to add this information in a data base. The final results show that were 69 dishes distributed in the following way: The municipality of centla obtain 40 rescued recipes and the municipality of Tacotalpa obtain 29 recipes and two of them were identifying in indigenous language as Zoque. The interaction between nature resources and the biocultural heritage for the municipality of centla they were mainly with the wildlife as crustaceans, chelonians, reptiles and fishes so for the mountain area Tacotalpa can be found plants, mammals, gasteropods and fishes.
\end{abstract}

Tourism, Rural, Culture, Education, Region

Citación: CARRILLO-CONTRERAS, Jaime, MAY-ARIAS, Elsi Margarita y MAZÓN-PÉREZ, Silvia Gertrudis. Patrimonio biocultural de Tabasco: elaboración de platillos tradicionales en comunidades indígenas. Revista de Sociología Contemporánea. 2019. 6-19: 7-15.

$\uparrow$ Investigador contribuyendo como primer autor. 


\section{Introducción}

México es considerado entre los primeros 4 países megadiversos (Sarukán, 2009: 10) por tener dos zonas biogeográficas (neartica y neotropical), la diversidad de plantas y animales que forman parte de la biodiversidad natural; y sin menospreciar la diversidad cultural que se ubica en los territorios indígenas con 68 lenguas diferentes (INALI, 2009:10), con sus propias prácticas culturales. Esto hace que en nuestra sociedad se tenga que reconocer que el patrimonio biocultural se encuentra precisamente en estos espacios donde confluyen todas estas características (Boege, 2008:16).

En Tabasco existen pueblos indígenas que aún conservan partes de ese patrimonio biocultural, pero que se ha visto disminuido por diversas causas, como proyectos de desarrollo que inciden en el cambio de los recursos naturales de la región, pérdida de población hablante de lengua indígena, actualmente en nuestro territorio se encuentran en mayor número hablantes de la lengua Yokot’an, $\mathrm{CH}^{\prime}$ ol y Tzotzil.

Precisamente en cuanto a la gastronomía heredada entre la prehispánica y la generada del encuentro cultural en la conquista; se obtuvo una mezcla de diversos platillos, que implican un conocimiento profundo de los recursos naturales, la continuidad de prácticas culturales para conservar olores, sabores, texturas en alimentos y acompañados de creencias que permiten en ocasiones la protección de la naturaleza, en muchos grupos indígenas.

Y como parte del conocimiento del patrimonio de los pueblos indígenas en Tabasco se encuentra aún en la gastronomía, y por lo tanto se busca que a partir de los platillos tradicionales que se preparan se manifiestan los conocimientos sobre las plantas y animales, la forma en que se utilizan y la asociación con alguna creencia que tiene una aplicación práctica simbólica. Además, en las poblaciones indígenas se han encontrado alimentos importantes para la subsistencia.
Por ello este proyecto buscó tener un registro de los platillos que se preparan en comunidades indígenas de Tabasco para reconocer los conocimientos que las personas tienen de los recursos naturales, las prácticas que realizan en relación a la gastronomía y las creencias que forman parte de su cosmos; finalmente dar propuestas de difusión del patrimonio biocultural para su revaloración y conservación.

\section{Materiales y métodos}

La metodología utilizada fue la cualitativa debido a que en este trabajo de investigación se requirió de describir el proceso de elaboración de los platillos y establecer el diálogo con personas al momento de realizar las entrevistas.

Selección de municipios. Los municipios con los que se trabajó corresponden a que en la Universidad Intercultural del Estado de Tabasco se oferta la licenciatura en Desarrollo turístico, el programa educativo cuenta con 13 años de trabajo en el municipio de Tacotalpa y en Centla 5 años, además considerando que son dos poblaciones que se encuentran opuestos en el límite norte-sur de Tabasco; y comparten el criterio de tener población indígena.

Municipio de Centla. Es uno de los municipios que se encuentra cercano a la línea de costa del Estado de Tabasco y ocupa el 10.9\% de la extensión estatal y cuenta con recursos acuáticos importantes que comparten la zona de la planicie como el río González y Laguna Santanita. La población total es de 110, 130 distribuidos en $50.4 \%$ de mujeres y el $49.6 \%$ de hombres; presenta el $4.09 \%$ de población que habla una lengua indígena de 3 años y más. Las viviendas alcanzan un total de 27500 y con respecto a los servicios de drenaje, servicio sanitario y electricidad por arriba del $90 \%$ y el que carece es el servicio de agua entubada con un $21.3 \%$ (INEGI, 2015:14).

Municipio de Tacotalpa. Es un municipio que tiene una porción de planicie y cuenta con zonas serranas con altitudes menores de 1000 msnm, ocupa el 3\% de la extensión estatal y tiene cuerpos acuáticos importantes como el Río de la Sierra y Puxcatán. 
La población total es de 48, 784 distribuidos en $49.8 \%$ de mujeres y $50.2 \%$ de hombres; presenta el $26.46 \%$ de población que habla una lengua indígena de 3 años y más.

Las viviendas alcanzan un total de 12 , 201 y en relación a los servicios de drenaje, servicio sanitario y electricidad por arriba del $90 \%$ y el más bajo es el servicio de agua entubada con $63.5 \%$ (INEGI, 2015:38).

Se utilizaron entrevistas abiertas, aplicadas a cocineras o cocineros tradicionales de la principalmente para documentar los diferentes platillos en los municipios de Centla y Tacotalpa, Tabasco; estos dos lugares se seleccionaron porque forman parte de las unidades académicas de la UIET.

En las entrevistas consideraron algunos datos personales y posteriormente el registro de los ingredientes, porciones y procedimiento; durante la intervención se trató de buscar las creencias relacionadas con los recursos naturales principales del platillo.

En algunos casos se recurrió a la observación participante (Álvarez-Gayou, 2003: 77) en el momento de la preparación de los platillos; donde se interactuó con las personas y dialogando sobre el proceso de elaboración del platillo.

La selección de cocineras se llevó a cabo con madres de familia o conocidas de los estudiantes de la licenciatura en Desarrollo Turístico a finales del año 2016 hasta el año 2018, privilegiando en el momento formativo y la vinculación con las comunidades de origen de donde proceden los estudiantes.

Las entrevistas fueron grabadas en un dispositivo móvil, se tomó fotografías de los platillos en algunos casos y posteriormente se transcribieron a un procesador de texto en el Observatorio turístico de la Universidad Intercultural del Estado de Tabasco. Por último, se agregaron a una base de datos la información generada de las cocineras tradicionales.

\section{Enfoque teórico}

A partir de considerar que en el modelo educativo en las Universidades Interculturales de México, se tiene por premisas el trabajo de vinculación con las comunidades las cuales se consideran funciones sustantivas y elemento clave (Moreno y Gómez, 2015: 8) y que el conocimiento o el saber de nuestros pueblos es determinante en el contexto de los estudiantes, sosteniendo el gran valor que se tiene tanto la diversidad natural como cultural, estos contribuyen a una mejor formación educativa (Casillas y Santini, 2006). De esa forma también la investigación es un pilar en las Interculturales, donde los estudiantes puedan emplear herramientas que les permita analizar su entorno; por ello este proyecto surge desde el aula hasta establecer el vínculo con las comunidades.

El alcance del proyecto se visualiza en este caso de forma espacial porque en principio identifica el registro de las cocineras tradicionales en comunidades indígenas de Tabasco, considerando que los platillos elaborados por estas personas son saberes que han sido heredados desde sus abuelos y que contiene el conocimiento sobre las plantas que se utilizan, los ingredientes y forma de captura de algunos organismos, que forman parte de su alimentación de manera frecuente o por temporadas.

Precisamente en la integración de los saberes, sus creencias y la práctica es lo que fundamenta el patrimonio biocultural de estos pueblos indígenas que con el paso de los años siguen conservando el legado histórico de nuestro país. Por ello a partir del 2010 la UNESCO nombra a la gastronomía mexicana como patrimonio de la humanidad por la diversidad de sabores y prácticas culturales existente en nuestro territorio.

A partir de esto se pretende que la gastronomía de Tabasco no solo se revalore por la riqueza de los recursos naturales; más bien por la riqueza cultural que se ha mantenido con el paso del tiempo y que se encuentra mayormente en las comunidades indígenas de nuestro estado. 


\section{Resultados y discusión}

Durante casi 2 años de trabajo en vinculación e investigación con estudiantes y docentes de la UIET, se tiene registrado un total de 45 cocineras y 4 cocineros distribuidos en los municipios de Centla y Tacotalpa, con un total de 69 platillos registrados (Tabla 1). Para el municipio de Centla se encontraron 29 cocineras con un total de 40 platillos y para Tacotalpa 16 cocineras, 4 cocineros con un total de 29 platillos.

\begin{tabular}{|c|c|c|}
\hline \multicolumn{3}{|c|}{ Platillos en el municipio de Centla } \\
\hline No & Nombre del platillo & Especie principal \\
\hline 1 & Armadillo asado & Dasypus novemcinctus \\
\hline 2 & Armadillo en verde & Dasypus novemcinctus \\
\hline 3 & $\begin{array}{l}\text { Camarón de popal con } \\
\text { yuca }\end{array}$ & Procambarus llamasi \\
\hline 4 & Camarón en verde & Procambarus llamasi \\
\hline 5 & Camarones con yuca & \\
\hline 6 & Camarones en puchero & \\
\hline 7 & $\begin{array}{l}\text { Camarones } \\
\text { enchipotlados }\end{array}$ & \\
\hline 8 & Camarones hervidos & Pomacea flagellata \\
\hline 9 & Caracoles sudados & Pomacea flagellata \\
\hline 10 & Ceviche de caracol & Pomacea flagellata \\
\hline 11 & Coctel de caracol & Pomacea flagellata \\
\hline 12 & Empanadas de camarón & \\
\hline 13 & $\begin{array}{l}\text { Empanadas de camarón } \\
\text { de popal }\end{array}$ & Procambarus llamasi \\
\hline 14 & Ensalada de camarón & \\
\hline 15 & $\begin{array}{l}\text { Hicotea con plátano } \\
\text { verde }\end{array}$ & Trachemys venusta \\
\hline 16 & Hicotea en sangre & Trachemys venusta \\
\hline 17 & $\begin{array}{l}\text { Huliche de camarón de } \\
\text { popal }\end{array}$ & Procambarus llamasi \\
\hline 18 & Iguana asada & Iguana iguana \\
\hline 19 & Iguana en adobo & Iguana iguana \\
\hline 20 & Iguana en tamal & Iguana iguana \\
\hline 21 & Iguana entomatada & Iguana iguana \\
\hline 22 & Lagarto en caldo & Crocodylus moreletii \\
\hline 23 & Lagarto entomatado & Crocodylus moreletii \\
\hline 24 & Mojarra asada & \\
\hline 25 & Mojarra empapelada & \\
\hline 26 & Pejelagarto en verde & Atractosteus tropicus \\
\hline 27 & Pochitoque en verde & $\begin{array}{l}\text { Kinosternon } \\
\text { leucostomum }\end{array}$ \\
\hline 28 & Puchero de res & \\
\hline 29 & Puscagua de pescado & \\
\hline 30 & Sábalo en caldo & Megalops atlanticus \\
\hline 31 & Sábalo en minilla & Megalops atlanticus \\
\hline 32 & $\begin{array}{l}\text { Sopa de camarón de } \\
\text { popal }\end{array}$ & Procambarus llamasi \\
\hline 33 & Sopa de caracol & Pomacea flagellata \\
\hline 34 & $\begin{array}{l}\text { Sopa de caracol con } \\
\text { calabaza }\end{array}$ & Pomacea flagellata \\
\hline 35 & $\begin{array}{l}\text { Tamal de camarón de } \\
\text { popal }\end{array}$ & Procambarus llamasi \\
\hline 36 & Tamalitos de chipilín & \begin{tabular}{|l|} 
Crotalaria \\
longirostrata \\
\end{tabular} \\
\hline 37 & $\begin{array}{l}\text { Tamalitos de frijol con } \\
\text { shis de chicharrón }\end{array}$ & \\
\hline 38 & Topen en mole & Dormitator maculatus \\
\hline
\end{tabular}

\begin{tabular}{|c|c|c|}
\hline 39 & Topen envuelto & Dormitator maculatus \\
\hline 40 & $\begin{array}{l}\text { Tortilla de yuca rellenas } \\
\text { de camarón }\end{array}$ & Manihot esculenta \\
\hline \multicolumn{3}{|c|}{ Platillos en el municipio de Tacotalpa } \\
\hline No & \begin{tabular}{|l|} 
Nombre del platillo \\
\end{tabular} & Especie principal \\
\hline 1 & Armadillo adobado & Dasypus novemcinctus \\
\hline 2 & Armadillo en puchero & Dasypus novemcinctus \\
\hline 3 & Armadillo frito & Dasypus novemcinctus \\
\hline 4 & Calabaza con tomate & Curcubita moschata \\
\hline 5 & Calabaza sudada & Curcubita moschata \\
\hline 6 & Caldo de joma con arroz & $\begin{array}{l}\text { Chamaedorea } \\
\text { tepejilote }\end{array}$ \\
\hline 7 & Caracol con frijol & Pachychilus chrysalis \\
\hline 8 & Caracol en blanco & Pachychilus chrysalis \\
\hline 9 & Caracol en rojo & Pachychilus chrysalis \\
\hline 10 & Caracol en verde & Pachychilus chrysalis \\
\hline 11 & Caracol sancochao & Pachychilus chrysalis \\
\hline 12 & Ceviche de caracol & Pachychilus chrysalis \\
\hline 13 & Chapaya con huevo & \begin{tabular}{|l|} 
Astrocarium \\
mexicanum
\end{tabular} \\
\hline 14 & Chaya con plátano & \begin{tabular}{|l|} 
Cnidoscolus \\
chayamansa
\end{tabular} \\
\hline 15 & Chirmol & \\
\hline 16 & Empanadas de chaya & $\begin{array}{l}\text { Cnidoscolus } \\
\text { chayamansa }\end{array}$ \\
\hline 17 & $\begin{array}{l}\text { Frijol con huevo } \\
\text { revuelto y plátano frito }\end{array}$ & \\
\hline 18 & Huevo con chaya & $\begin{array}{l}\text { Cnidoscolus } \\
\text { chayamansa }\end{array}$ \\
\hline 19 & Pochitoque en verde & $\begin{array}{l}\text { Kinosternon } \\
\text { leucostomum }\end{array}$ \\
\hline 20 & Puchero de pollo criollo & gallus \\
\hline 21 & Pushane o niño envuelto & \\
\hline 22 & Pux & \\
\hline 23 & Sardina en mone & \\
\hline 24 & $\begin{array}{l}\text { Sopa de arroz con } \\
\text { armadillo }\end{array}$ & Dasypus novemcinctus \\
\hline 25 & $\begin{array}{l}\text { Tamal de caracol con } \\
\text { frijol }\end{array}$ & Pachychilus chrysalis \\
\hline 26 & $\begin{array}{l}\text { Tamal de masa con } \\
\text { caracol }\end{array}$ & Pachychilus chrysalis \\
\hline 27 & $\begin{array}{l}\text { Tamalito de chaya con } \\
\text { tomate }\end{array}$ & $\begin{array}{l}\text { Cnidoscolus } \\
\text { chayamansa }\end{array}$ \\
\hline 28 & Tamalito de yuca & Manihot esculenta \\
\hline 29 & Tortitas de yuca & Manihot esculenta \\
\hline
\end{tabular}

Tabla 1 Platillos identificados en los municipios de Centla y Tacotalpa, Tabasco

Se determinó que de la mayoría de las cocineras del municipio de Centla muy pocas entienden la lengua indígena y por lo tanto no la hablan, en este caso nos referimos a la lengua Yokota`n; sin embargo, para el municipio de Tacotalpa la mayoría entiende y habla la lengua indígena Ch`ol. Estos resultados evidencian por origen por el proceso de desarrollo del estado de Tabasco. 
En Tacotalpa existen platillos que se nombran en la lengua Zoque por ejemplo (Pushane o niño envuelto y Pux) por lo contrario en Centla no se identificó alguno en lengua Yokotan. Con respecto al género en el municipio de Centla y Tacotalpa mayormente los hombres se encargan de conseguir las especies mediante diferentes técnicas de captura dependiendo de la zona.

Las mujeres se encargan principalmente de la preparación de los platillos y conseguir los ingredientes principales, los cuales se obtienen mayormente del traspatio, donde existen muchas especies vegetales. Por lo tanto, como sugiere Fraire, 2017:9 que la producción agroecológica de los huertos de traspatios son opciones para satisfacer parte de las necesidades alimentarias en las familias tabasqueñas e importantes para la conservación de la biodiversidad con alto valor cultural.

Es evidente que aún existen los roles por género, sin embargo, para Tacotalpa se encontró que existen hombres que preparan sus platillos; aunque no se descarta que en Centla también exista esta situación.

Con respecto a los ingredientes para la preparación o acompañamiento de diversos platillos en los dos municipios, aunque no es explicito, al menos uno de los ingredientes básicos y considerados prehispánicos forma parte del platillo, en estos casos hablamos de chile, frijol y maíz.

Es por lo anterior que la UNESCO declaró a la gastronomía mexicana en el 2010 como patrimonio de la humanidad porque representa un legado histórico diverso lleno de conocimientos, prácticas y creencias que aún se conservan. El conocimiento indígena como señala Toledo y Barrera 2008, es holístico porque se encuentra fuertemente apegado a las prácticas sobre el uso y manejo de los ecosistemas que se realizan en las comunidades indígenas para resolver necesidades prácticas que se presentan en un espacio. Este conocimiento se basa en la tradición oral (Maldonado y Díaz, 2010: Pág. 65) y (Centurión, et al 2003: p. 90) como forma de transmisión de esos conocimientos que han estado por muchas generaciones y que tiene dos dimensiones esenciales que son el tiempo y el espacio.
Esos conocimientos tradicionales están asociados a los agroecosistemas, conformado por un fino conocimiento físico y biótico de su medio local como señala Beltrán y Huicochea, 2010: 124) y que está respaldado por las habilidades prácticas, creencias y valores que le dan identidad a las familias campesinas.

Basados en lo anterior en el estado de Tabasco se logran identificar dos momentos importantes desde la parte ecológica que comprende la época de secas (marzo-agosto) y de lluvias (septiembre-febrero). Las personas aprovechan estas temporadas para poder obtener diversos alimentos que consumen en el caso de Centla, el Topen Dormitator maculatus, pejelagarto Atractosteus tropicus y el caracol Pomacea flagellata que aparecen al inicio de las lluvias intensas en el mes de noviembre (aprovechan diversas corrientes de aguas) y como consecuencia de las inundaciones en el mes de febrero se aprovecha el camarón de bajo o de popal Procambarus llamasi como señalan los pobladores y se cocina de diferentes formas como se ha encontrado en este proyecto y se prepara en tamal, en verde o con yuca (Figura 1).

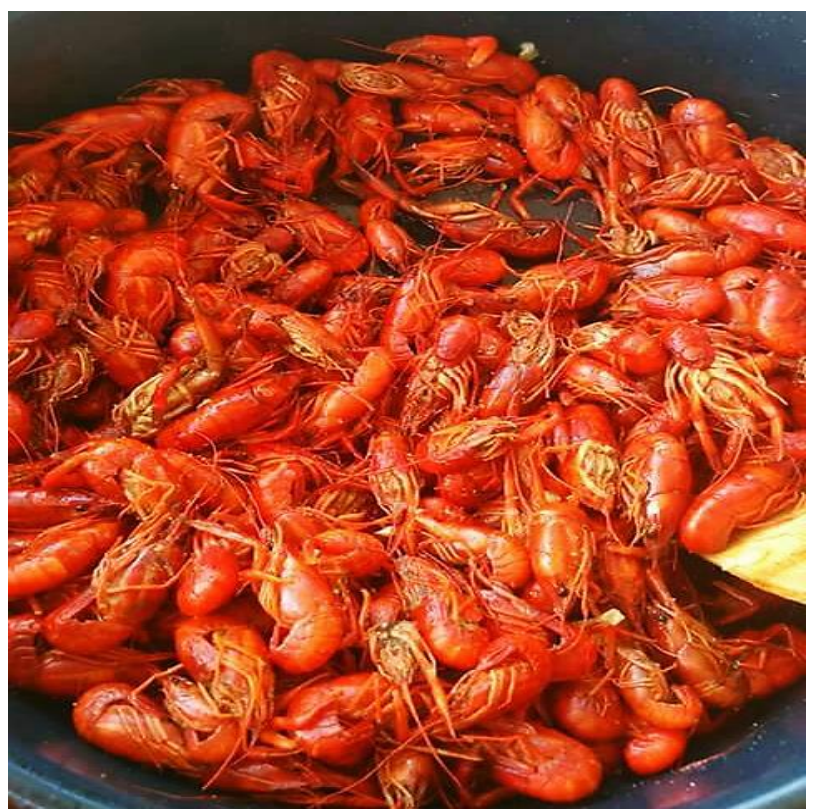

Figura 1 Camarón de popal P. llamasi, preparado en el municipio de Centla, Tabasco 
Desde una mirada occidental, existen personas que aprovechan estas temporadas para incrementar la economía, se ha encontrado (visitas en el centro de Villa Vicente Guerrero, Centla) que en el caso del Topen a inicios de su aparición presenta elevados precios de venta por kilogramo llegando a $\$ 60.00$ pesos; en medida que la oferta aumenta el precio va disminuyendo en aproximadamente una semana logra tener hasta $\$ 35.00$ pesos por kilogramo. Esta especie es muy consumida en las zonas bajas de Tabasco.

Aunque en esta investigación no se tenga determinado el uso de chile amashito en cada una de los platillos, si es importante mencionar que es muy consumido en Tabasco (González, 2017: 3), para acompañar los platillos en Centla como empanadas, pejelagarto asado, coctel de camarón y en Tacotalpa se ha encontrado grupos de campesinos que utilizan el chile amashito para acompañar el pozol en el receso de trabajo en el campo. Por lo tanto, el mismo autor menciona que es importante mantener la agricultura familiar en varios países porque los alimentos se producen en forma ecológica (González, 2017: 7).

Para el caso del municipio de Tacotalpa, los campesinos también tienen conocimientos sobre las temporadas diferenciales en el estado; solo que el aprovechamiento está más asociado a los recursos naturales que encuentran en las montañas (tipo de vegetación selva mediana perennifolia-arroyos o río). Por ejemplo, la caza de especies de mamíferos es una práctica que requiere muchos conocimientos y que ellos consideran (no cualquiera es cazador). Con respecto a varios platillos elaborados uno de los más representativos son la Chapaya Astrocarium mexicanum y el Caracol Pachychilus crysalis (Figura 2), el primero corresponde a un tipo de palma que en los meses de marzo-abril comienza a tener la inflorescencia y está es aprovechada por los pobladores, además que está relacionada con la creencia de no poder comer carne por encontrarse en cuaresma. Esto también lo comenta Centurión, et. al., 2003, reportando que además son recolectores de los pocos relictos de selva que se encuentran y que sobresalen varios tipos de palmas pero que existen otras especies que se consumen.

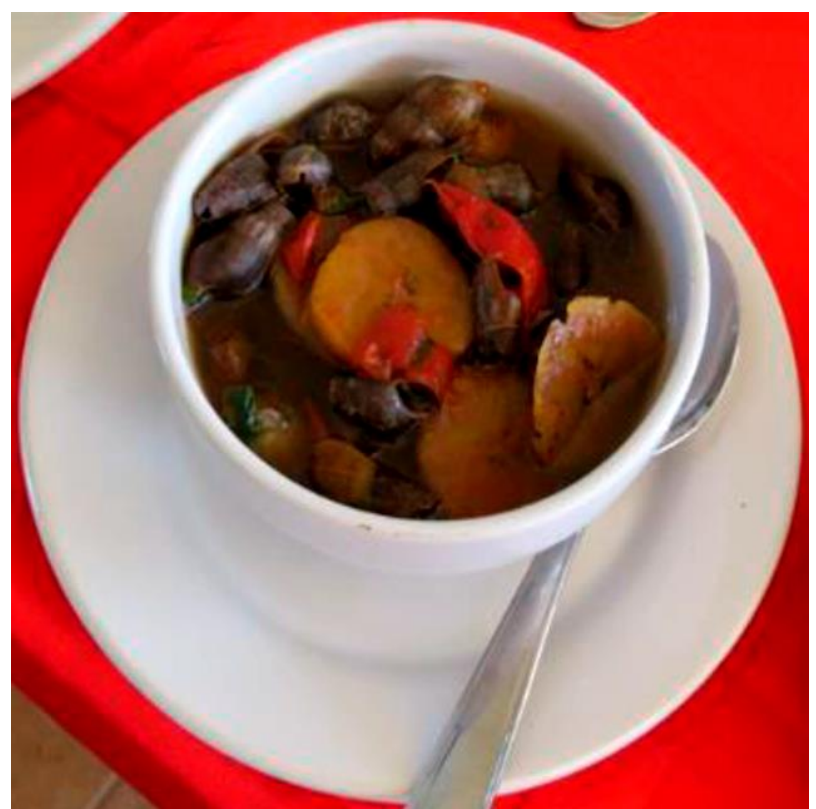

Figura 2 Caracol guisado, P. crysalis. Tacotalpa, Tabasco Fuente: muestra gastronómica 2017

También se ha observado que la chapaya se encuentra en mercados de la capital del estado y que se vende especialmente como alimento de comunidades indígenas. Con respecto al caracol se ha documentado en Nicaragua (López y Urcoyo, 2012) y recientemente con las personas en el poblado Oxolotán consideran que consumir alimento natural refiriéndose al caracol (Morales, 2017:19), les da una mejor esperanza de vida "que esto es bueno para el cuerpo" según (Carrillo et al 2017).

En muchas zonas de Tabasco la milpa es un agroecosistema como menciona (Morales et al; 2015: 39) muy utilizado por campesinos; al menos en las comunidades de Tacotalpa: La Pila, Cuviac, Tomas Garrido, y Oxolotán se determinó que la milpa se establece en dos ciclos de cultivo: primavera-verano (mayo-junio), conocida como milpa de año, y otoño-invierno, conocido como tornamil (noviembrediciembre). Además, que la milpa se diversifica con frijol, calabaza y chile.

Por lo tanto, en relación a la gastronomía se interrelaciona los espacios donde las personas capturan o cortan las especies que utilizan para preparar sus alimentos; así como los tiempos en los cuales hay especies particulares que están asociadas a la creencia religiosa o que se relaciona con alguna fiesta. 
Con respecto a las creencias en el municipio de Centla se mencionó que cuando las personas van a salir a capturar especies silvestres para la preparación de alimentos no se debe mencionar, porque existe una consecuencia, la cual consiste en que no se tenga una buena captura de especies o nula. En Tacotalpa, las creencias con respecto cuando se sale a cazar, no se debe ir haciendo ruido o silbando porque también no se podrá obtener algunas especies para alimentos. Algunas especies solo se pueden consumir en temporada de cuaresma, por la creencia de que dios no mandará bendiciones a las familias por consumir carne, principalmente se consume pescado y camarón en el municipio de Centla y en Tacotalpa se consume la inflorescencia de la Chapaya (Figura 3).

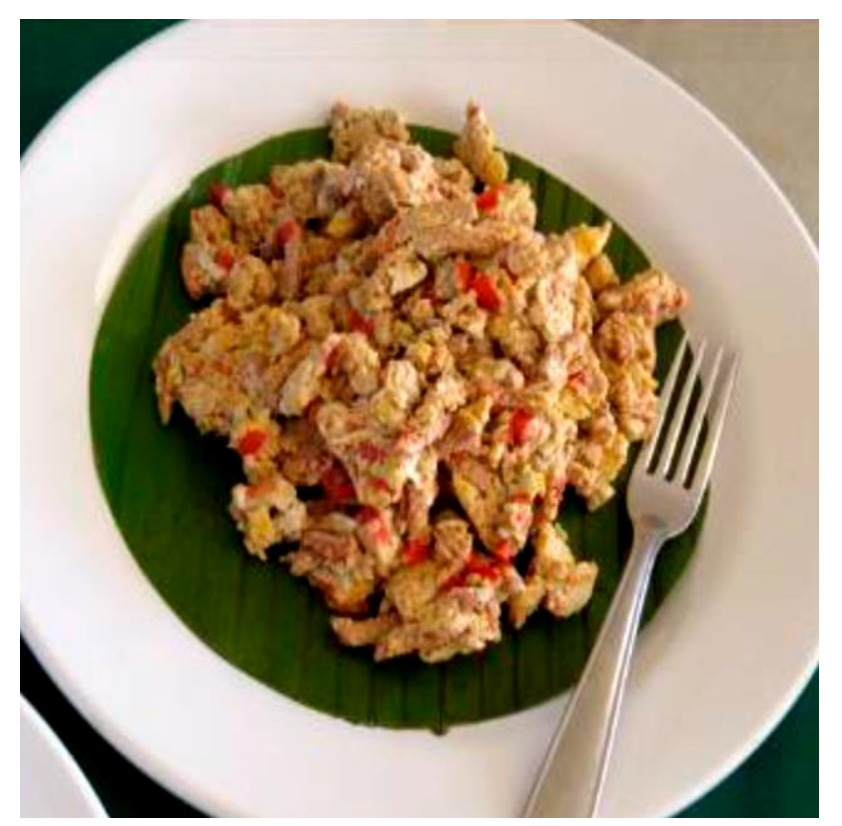

Figura 3 Chapaya con huevo, A. mexicanum. Tacotalpa, Tabasco

Fuente: muestra gastronómica 2017

En la sierra de Tacotalpa se ha encontrado que las creencias están relacionadas con la protección de animales considerados como amenaza, como en el Ejido Noypac (Maldonado y Díaz, 2010).

La obtención de alimentos y preparación de platillos tiene relación con las creencias, en Centla las personas tienen que prepararlos porque los santos difuntos llegan a la mesa a comer el platillo que consumían o les gustaba; por ello se preparan en ciertas festividades o conmemoración: día de muertos el Uliche, se prepara con pollo, es considerado de origen indígena y prehispánico.
Una de las creencias remarcadas en ambos municipios tiene relación con el cuidado al momento de ir al campo, y que es muy comentada sobre las serpientes venenosas que al momento en que las personas que tienen relación con un embarazo, en este caso al conyugue la serpiente no lo puede atacar.

La mayoría de las recetas de los dos municipios en sus ingredientes principales se utilizan especies de plantas y animales silvestres, esto representa que el patrimonio biocultural de estas zonas continúa manifestándose en la alimentación de estas comunidades indígenas, pero en el municipio de Centla está más acentuada la pérdida de un elemento cultural como es la lengua. Tabasco no cuenta con algún plan estratégico que permita incidir en la conservación del patrimonio biocultural.

En el caso de Tacotalpa se pudo observar la forma en la cual se lleva a cabo el proceso de preparación de 8 platillos con una especie de caracol $P$. crysalis. Es una práctica que en muchas de las comunidades cercas acostumbran a comer. En el caso de Centla se ha encontrado que existen varias especies de quelonios que se consumen en las comunidades; para ello se visitó con estudiantes el momento de preparación de hicotea en sangre Trachemys venusta (Figura 4); es uno de los ingredientes prehispánicos.

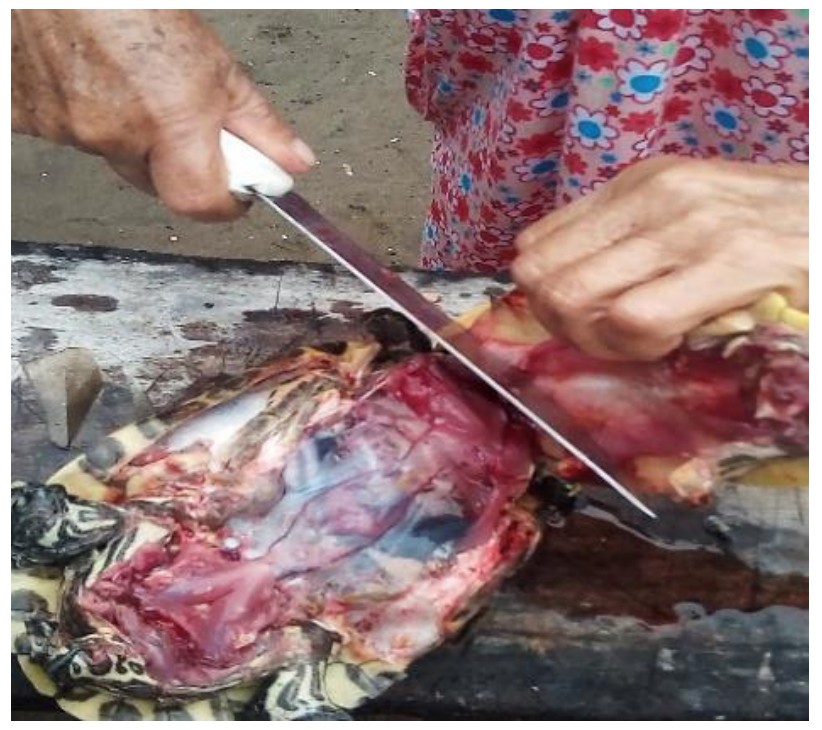

Figura 4 Municipio de Centla: Doña Apolonia, corte de la hicotea 


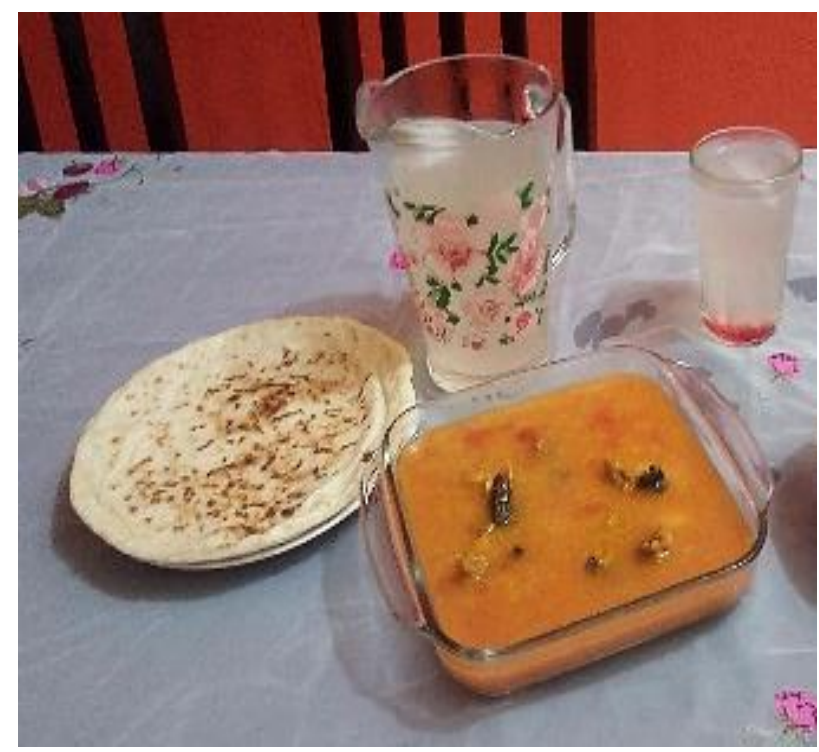

Figura 5 Municipio de Centla: Doña Apolonia, platillo preparado

Existe una relación muy estrecha entre los conocimientos que las personas tienen sobre los recursos naturales (épocas de captura, biología de las especies, anatomía, diferencias entre especies, técnicas de captura, ecología), con las prácticas asociadas en cada contexto; en Centla están asociadas mayormente al agua (ríos, lagunas y zonas inundables) en el caso de la Sierra de Tabasco, las practicas están ligadas al Río, montaña y selva). Actualmente los grupos de Choles cubren una buena parte del territorio en Tacotalpa; aunque no sean los grupos que vivieron originalmente como los de origen ore (zoque) en Oxolotán (Rojas et al., 2015: 16). La gastronomía tabasqueña tiene mucha importancia por su tradición alimentaria y como en muchos otros pueblos como señala Beltrán y Huicochea, 2010: 72, forman parte de la historia y son elementos del patrimonio cultural. Con respecto a las creencias en las zonas de la planicie Tabasqueña proviene de los dueños del agua y en la serranía se concibe como los dueños del monte o montaña.

\section{Conclusiones}

Se registraron un total de 69 platillos incluyendo los dos municipios (Centla y Tacotalpa) en Tabasco. Las cocineras que se registraron en total corresponden a 49, correspondiendo 29 al municipio de Centla y 20 a Tacotalpa. En términos de patrimonio es mayor la presencia de los hablantes de lengua indígena $\mathrm{CH}^{\circ}$ ol para Tacotalpa; sin embargo, para el municipio de Centla no se registró ninguna cocinera hablante de lengua Yokot’an.
Se encontró que las personas identifican bien los tiempos y espacios para la captura de especies en los dos municipios y que estos tienen relación con la preparación de platillos tradicionales en tiempos específicos; tanto asociados a la creencia religiosa como aprovechamiento por temporadas que ayudan a la alimentación familiar.

\section{Referencias}

Álvarez-Gayou, J. J. L. (2003). Cómo hacer investigación cualitativa. Fundamentos y metodología. Edit. Paidós Educador. México, D. F. 222 p.

Boege-Schmidt, E. (2008). El Patrimonio Biocultural de los Pueblos Indígenas de México. Instituto Nacional de Antropología e Historia y Comisión Nacional para el Desarrollo de los Pueblos Indígenas. México.344 p.

Carrillo, Contreras Jaime; De los Santos, Ruiz Cynthia Paola; Reyes, Cruz Emma y Ramírez, Méndez Roberto Edi. (2017). Saberes de la cultura $\mathrm{CH}$ 'ol sobre los caracoles acuáticos en la Sierra de Tabasco. Revista Transdisciplinaria de Estudios Migratorios. Vol.3 (9): 17-25.

Casillas Muñoz, María de L. y Santini, Villar L. (2006). Universidad intercultural Modelo Educativo. Coordinación General de Educación Intercultural y Bilingûe. México, D. F.

Centurión, Hidalgo D.; Cázares, C. J. G.; Espinoza, Moreno. J. Poot Matu, J. E.; Mijangos, C. M. A. (2003). Aprovechamiento alimentario de inflorescencias en la región sierra del estado de Tabasco. Polibotánica. No. 15: 89-97.

Centurión, Hidalgo D., Alor, Chávez M. J., Espinoza, Moreno J., Gómez, García E., Solano, M. L. y Poot, Matu J. E. (2009). Contenido nutricional de inflorescencia de palmas en la sierra del estado de Tabasco. Universidad y $\begin{array}{llll}\text { Ciencia. } & 25 & \text { (3): } & \text { 193-199. }\end{array}$ www.ujat.mx/publicaciones/uciencia.

Fraire, S. L. (2017). La mujer tabasqueña: clave para la producción agroecológica familiar. Diálogos del Consejo de Ciencia y Tecnología del Estado de Tabasco: 6-9 pág. 
González, C. N. (2017). Chile Amashito: Sabor picante de Tabasco. Diálogos del Consejo de Ciencia y Tecnología del Estado de Tabasco. $51: 5$

Instituto Nacional de Lenguas Indígenas. (2009). Catálogo de las lenguas indígenas nacionales. Variantes lingüísticas de México con sus autodenominaciones y referencias geoestadísticas. México, D. F. 371p.

López, S. J. A. y Urcoyo, J. (2012). First report of Faunus ater (Pachychilidae: Gastropod) on the American Continent: A mature empty specimen from Masachapa, Nicaragua, Central America. Research Journal of the Costa Rican Distance Education University. 4 (2): 187-189.

Maldonado Cano Daniela y Díaz Ramírez Alicia. (2010). Paisajes e identidad en la sierra de Tabasco. Península, vol. V: (2).

Morales, V. G., Mariaca, M. R. y Padilla V. J. (2015). Tecnología campesina en la milpa de ladera en Tacotalpa, Tabasco. 33-51. In Moreno, U. V. y Gómez V. E. M. Problemáticas del Sur. Antología. Universidad Intercultural del Estado de Tabasco. México.

Morales, V. G. (2017). El potencial turístico de Tacotalpa: Sistemas agroecológicos. Diálogos del Consejo de Ciencia y Tecnología del Estado de Tabasco: 14-19 pág.

Moreno, U. V. y Gómez V. E. M. (2015). Antología. Investigación vinculada en las Universidades Interculturales de México. Antología. Universidad Intercultural del Estado de Tabasco. México. 147 p.

Rojas, L. V. R., Navarro, M. S. I. y Escobar, P. A. (2015). Historia de la UIET, su génesis y situación actual. Saberes de los pueblos, la UIET a diez años. I (1): pág. 16.

Sarukán, J. et al. (2009). Capital natural de México. Síntesis: conocimiento actual, evaluación y perspectivas de sustentabilidad. Comisión Nacional para el Conocimiento y Uso de la Biodiversidad. México.

Toledo, Víctor M. y Narciso Barrera Bassols. (2008). La memoria biocultural. La importancia ecológica de las sabidurías tradicionales. 232 p. Perspectivas agroecológicas, Junta de Andalucía Icaria Editorial, Barcelona.
Vadillo, L. C. Antropología e historia de la diversidad cultural de la alimentación en Campeche. In Huicochea, G. L. y Cahuich, C. M. B. (2010). Patrimonio biocultural de Campeche. Experiencias, saberes y prácticas desde la antropología y la historia. El colegio de la Frontera Sur. Chiapas, México. 247 p. 